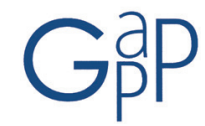

GESTIÓN Y ANÁLISIS DE POLÍTICAS PÚBLICAS, Nueva Época - N.º 15, enero-junio 2016 - ISSN: 1989-8991

DOI: http://dx.doi.org/10.24965/gapp.voi15.10311

Agustín García Inda

Cuerpo Superior de Administradores Civiles del Estado

aginda@gmail.com

\title{
Hamburguesas y eficiencia. Del estado de bienestar a la sociedad del bienestar
}

\section{Resumen}

La crisis iniciada en 2008 ha mostrado una serie de problemas estructurales en distintos planos del sistema económico global y, en particular, en la sociedad española. Las crisis primero 'subprime' norteamericana, de la zona euro después, de la burbuja inmobiliaria, etc. en España ha mostrado algunas contradicciones, especialmente en la formulación y provisión del bienestar social.

En esta comunicación se presenta la llamada "metáfora de la hamburguesa" como constructo teórico que explica las ineficiencias del sector público y cómo, por sus características políticas y sociales, fracasan las soluciones. Ese fracaso, las tendencias demográficas y los déficits crónicos exigen respuestas que garanticen los derechos políticos, civiles y sociales. Las soluciones se resumen en la "teoría de las tres eses": Suprimir iniciativas públicas, Sustituir público por privado y Simplificar; en definitiva, aplicar el principio de subsidiariedad hasta el individuo para pasar del Estado de Bienestar a la Sociedad de Bienestar.

Para ello se plantean, -considerando la literatura 'clásica' sobre las políticas sociales, el estado social, los mundos del bienestar, el postcapitalismo y el pluralismo del bienestar- tres preguntas: (i). ¿Qué ineficiencias se dan en las políticas públicas y sociales? (ii). ¿Puede la sociedad española en el contexto de la Unión Europea soportar esa ineficiencia cronificada? (iii). ¿Sirve la respuesta al modo holandés tal como se expresó en el discurso del príncipe de septiembre de 2013?

Los condicionantes socio-políticos del sector público y sus consecuencias económicas determinan su ineficiencia intrínseca. El propio sistema obstaculiza su solución, dada la preferencia del político por el rédito electoral. Los modelos de empleo y gasto maniatan al gestor y otros factores socioculturales impiden la reforma. Diversos planes de modernización, medidas de simplificación administrativa y de administración electrónica, etc. no han tenido efecto sobre la eficiencia.

Con la Teoría de las 35 se propone un proceso de reflexión y "adelgazamiento" competencial para que la subsidiariedad del Estado respecto a la sociedad civil sea real y efectiva, sin renunciar al Bienestar. La provisión de Bienestar social habrá de venir por otro camino que conducirá a la Sociedad del Bienestar. Individuos, asociaciones o empresas han de recuperar "la res publica". Se trata de equilibrar el actual trade off de responsabilidades: la responsabilidad social "activa" (de implicación) del sector privado y del llamado "tercer sector" y la responsabilidad "pasiva" (de autolimitación) del Sector Público.

Palabras clave

Estado, Bienestar, Sociedad Civil, Administración, Eficiencia

\section{Burgers and efficiency. Welfare state to the welfare society}

\section{Abstract}

The crisis that began in 2008 has shown a number of structural problems at different levels of the global economic system and, in particular in Spanish society. The American 'subprime' crisis first, and then the Eurozone crisis, the real estate bubble, etc., have shown some contradictions in Spain, especially in the development and provision of social welfare.

This article introduces the so called "Burger's metaphor" as a theoretical construct that explains the inefficiencies of the public sector and how the solutions fail because of their political and social characteristics. That failure, the demographic trends and the chronic deficits demand answers that guarantee political, civil and social rights. The solutions are summarized in the " $3 S$ Theory": Suppressing public initiatives, Superseding public by private and Simplifying; that is, applying the principle of subsidiarity to the individual to be able to move from Welfare State to Welfare Society.

In order to get this -considering the 'classical' literature on social policies, social status, the worlds of welfare, post-capitalism and pluralism of welfare-three questions arise: (i). What inefficiencies exist in public and social policies? (ii). Can Spanish society in the European Union context bear this chronic inefficiency? (iii). Does the Dutch way serve as an answer, as it was expressed in Prince's speech on September 2013?

The socio-political determinants in the public sector and its economic consequences cause its intrinsic inefficiency. The system itself hinders the solution, given the politician's preference for the electoral revenue. Employment and spending patterns hobble the manager, and other sociocultural factors prevent the reform. Several modernization plans, administrative simplification programs and e-government actions etc. have not had any effect on efficiency.

"3S Theory" proposes a process of reflection and "competencies' thinning", so the State's subsidiarity towards civil society becomes real and effective, without sacrificing Welfare. The supply of social welfare will have to come from a different way that will lead to the Welfare Society. Individuals, associations or companies must recover the "res publica". It is about balancing the current responsibilities' trade off: "Active" social responsibility (of engagement) of private sector and the so-called "third sector" and "passive" responsibility (Self-limiting) of public sector.

Keywords

State, Welfare, Civil Society, Public administration, Efficiency 


\section{INTRODUCCIÓN}

"From cradle to grave", proclamó en 1941 el Informe Beveridge para consagrar el principio de cobertura universal de la seguridad social para todos los ciudadanos. Desde entonces, el Estado de Bienestar (EB, en adelante) ha estado sometido a una crítica permanente; solo 3 años después del citado Informe, Hayek publicaba Road to Serfdom y también desde la izquierda, aunque con otros argumentos, llegarían duras críticas (Saville, Wedderburn, Baran y Sweezy, Offe y Miliban, por ejemplo).

La crisis económica de los 70 puso de relieve los problemas económicos, de gobierno y fiscales que generaba el EB y la consiguiente crisis de legitimidad lo que provocó que todos los países introdujeran reformas más o menos avanzadas. Lo mismo ha sucedido con la crisis de 2008 , que ha vuelto a mostrar la insostenibilidad que supone la estadística 7/25/50 y la necesidad de nuevas reformas. El príncipe de Holanda, en su discurso de 2013 fue muy explícito. "Como consecuencia de las tendencias sociales, como el envejecimiento de la población y la internacionalización, nuestro mercado de trabajo y el sistema de servicios públicos no satisface la demanda del siglo $\mathrm{XXI}$...debemos reconocer que los servicios públicos y los esquemas precisan ser ajustados..., el clásico estado del bienestar de la postguerra produjo esquemas que no son sostenibles en su forma actual y que ya no cumplen las expectativas de la gente. En el mundo actual, la gente quiere tener la posibilidad de decidir por sí mismos, dirigir sus propias vidas y cuidarse unos a otros. Es en este desarrollo que los servicios de seguridad social y sanitarios se organizarán cerca de la gente y de una manera coherente..."

En este contexto de revisión y reforma, el presente artículo invita a un enfoque realista, fruto de la constatación histórica de que las circunstancias (políticas, económicas, jurídicas y sociales) que condicionan la decisión política y la gestión pública derivada de dicha decisión, provocan la ineficiencia sistémica del sector público. Para corregir esa ineficiencia se han puesto en marcha distintas soluciones que, sin embargo, no logran evitar la ineficiencia como defecto estructural. Las tendencias demográficas y los déficits presupuestarios continuados en las economías occidentales, unido a la ausencia de soluciones reales y permanentes imponen un enfoque integrador, un nuevo pacto social (Moreno, 2000) articulado sobre renovadas bases morales (solidaridad), económicas (sostenibilidad) y políticas (legitimidad); en definitiva, un cambio de modelo que sin dejar de asegurar unos derechos sociales básicos, "despolitice" o "despublifique" el EB hacia una "Sociedad de Bienestar", reduzca el peso del sector público y huya de la gestión pública en producción de bienes y servicios públicos: ese cambio es el que se propone con la "Teoría de las 3S" (3S, en adelante).

\section{LAS INEFICIENCIAS EN EL DESARROLLO DE LAS POLÍTICAS PÚBLICAS}

Imagínese que tras una dura mañana de trabajo y sin apenas haber desayunado, necesita comer algo. Lo que más le apetece es una gran hamburguesa; por ello, decide ir a un cercano restaurante de comida rápida en el que, por diez euros, podrá tomar una hamburguesa con patatas que saciará su apetito antes de continuar su jornada laboral.

Este sencillo ejemplo sirve para ilustrar las fases en que se generan los riesgos de ineficiencia en cualquier actividad productiva de bienes y servicios. En el caso de la hamburguesa, existe una necesidad (apetito y recuperar energía) por lo que a cambio de 10 euros (inputs) se adquiere una hamburguesa (output) con la que se espera satisfacer aquellas necesidades; es decir, disfrutar de su sabor a la vez que recuperar energía (outcomes), aunque pueda subir algo el colesterol (externalidades negativas).

También en el proceso de producción de bienes y servicios por el Estado² o en su actividad redistributiva (de rentas monetarias) se reproduce ese esquema que mostrará los posibles fallos de eficiencia:

\section{a) Ineficiencias de origen}

Antes de ir a la hamburguesería debería preguntarme si realmente tengo hambre, si necesito comer o es un capricho de mi glotonería y podría esperar. Suponiendo que realmente mis niveles de glucosa estén por los suelos y necesite el aporte calórico de la deseada hamburguesa me preguntaré si no era mejor haber desayunado más o traer un sándwich de casa. Algo parecido debería plantearse el decisor político. ¿Es necesaria la intervención pública?, ¿es prescindible o presta ya alguien (público o privado) ese servicio?. Esto nos lleva a un análisis de las ajenidades y las duplicidades.

1 Significa, que, en términos aproximados, Europa representa al mismo tiempo el $7 \%$ de la población mundial, el $25 \%$ del PIB y el $50 \%$ del gasto público mundial.

2 Aunque son evidentes las diferencias conceptuales de los términos "Estado", “Administración" o "Sector Público", en este artículo se ha ce un uso indistinto pues lo relevantes es la consideración de cualquier poder público o Administración territorial o institucional como unidades de intervención social y de gasto público. 
Lo primero (ajenidades) es todo aquello que hace la Administración que no es (o no debe ser) propio de ella, lo que nos lleva a la gran pregunta: ¿Para qué está el Estado?. Esta es la pregunta que anida "en el corazón de un viejo debate (Micklethwait y Wooldridge, 2015), un debate que desapareció durante la fase del "todo lo que VD pueda comer" de la democracia moderna"' Desde que los liberales clásicos pensasen en un Estado limitado a las funciones básicas que asegurasen su existencia y el cumplimiento de la Ley, el Estado no ha dejado de crecer hasta convertirse (King, A. 1975) en "una suerte de compañía de seguros de responsabilidad ilimitada en el negocio de asegurar a todas las personas, en todos los tiempos, contra cualquier riesgo concebible", un EB del que ni Bismark, ni Beveridge, ni siquiera Keynes, reconocerían su alcance actual. Y "Cuantas más responsabilidades asume Leviatán, peor las gestiona y más gente se indigna, lo que sólo hace que aún pidan más ayuda. Éste es el círculo vicioso de las políticas progresistas".

Por ello y por razones de sostenibilidad es necesario que los gobiernos se limiten a ejecutar aquellas iniciativas que le son propias y no puedan ser realizadas por el sector privado (lucrativo o no lucrativo) y que vayan a tener un impacto real directo. Un exceso de intervención provoca una "ineficiencia de origen" o "ineficiencia total"; ejemplos de ello son los inútiles observatorios o los "aeropuertos sin aviones".

Menos difícil que identificar las ajenidades es identificar "las duplicidades" tanto con el sector público como con el privado. Basta mirar el Informe de la Comisión para la Reforma de las Administraciones Públicas (CORA) para constatar la repetición de órganos con idénticas funciones, casi todas ellas derivadas de la estructura territorial de Estado (observatorios, agencias de evaluación universitaria, defensores del pueblo...). Por un "efecto imitación" cualquier gobierno no quiere "ser menos" que su vecino y tiende a copiar órganos y funciones. Otra razón es la tendencia a mantener y nunca eliminar, lo que lleva en muchas ocasiones a sostener servicios paralelos, como la subvención a trenes sin pasajeros que hacen las mismas rutas que hacen autobuses regulares bajo concesión pública (también subvencionada).

Las duplicidades con el sector privado se producen cuando el servicio público ya es (o puede ser) prestado por el sector privado. Ejemplo de ello son las escuelas municipales de educación infantil "0-3 años". Si lo que el ayuntamiento desea es favorecer la conciliación familiar o que niños sin recursos puedan ir a escuelas privadas, podría aprovechar la "capacidad instalada" (aunque sea privada) y dar becas o ayudas o negociar un Convenio de colaboración con la guardería privada.

A estas ineficiencias de origen o "totales" (ejecución de ajenidades y duplicidades) hay que añadir otras derivadas del momento de la toma de decisión y su ejecución. Estas ineficiencias acompañarán hasta el final a toda la actuación o política pública: los retardos económicos. La teoría de los retardos explica que existen una serie de desfases que disminuyen los efectos de una política. En primer lugar puede darse un retardo de reconocimiento (recognition lag), tiempo que transcurre desde que existe el problema hasta que se observan cambios en las variables económicas que requieren reformas. El tiempo transcurrido desde que se reconoce la necesidad de esas reformas hasta que se adoptan (análisis, discusión parlamentaria, aprobación...) supondrá un retardo de acción (action lag). Y finalmente, el retardo externo (effect lag) será el desfase producido desde que se activan las reformas hasta que se muestran sus efectos ${ }^{3}$.

\section{b) Ineficiencias económicas input-output}

Si por los 10 euros nos dan una magnífica hamburguesa con unas sabrosas patatas quedaremos satisfechos y volveremos a ese restaurante. Es la relación calidad/precio, la ineficiencia puramente económica que se deriva de la relación entre el producto o resultado inmediato (output) y los recursos materiales, humanos y financieros (inputs) empleados. Cuando el responsable político considera oportuna una iniciativa debería plantearse cuál es el modo en que menos inputs sean necesarios para conseguir los resultados esperados o cómo puede obtener más outputs con los mismos inputs (productividad).

Si antes la pregunta era ¿Tengo que actuar?, ahora la pregunta será ¿Tengo que actuar yo o lo hace o puede hacer otro?; en otras palabras: ¿Pueden hacer el sector privado aquello que el sector público quiere que se haga?, ¿tiene el estado el monopolio de lo público? Y estas preguntas nos las haremos porque, como se verá en el siguiente apartado, los procesos de producción de bienes y servicios públicos o de redistribución de rentas (transferencias monetarias) son una fuente inagotable de ineficiencias a causa de los sistemas de contratación, de gasto y de empleo públicos que obligan a destinar una gran cantidad de recursos.

3 Ejemplo de ello es la adopción de medidas "anticrisis" de los Gobiernos de Zapatero y Rajoy en 2011-2012. Zapatero tarda en reconocer la crisis (meses perdidos) y en adoptar medidas (más meses perdidos); por otro lado, los nuevos gobiernos autonómicos (tras las elecciones de mayo de 2015) a la vista de las siguientes elecciones generales de finales del mismo año no parecen tomar medidas hasta que éstas se celebran (más meses perdidos). 


\section{c) Ineficiencias finales (outcomes)}

Siguiendo con nuestro ejemplo, hoy no había desayunado, mi glucosa estaba baja y necesitaba el aporte proteínico de la hamburguesa y los hidratos de las patatas así que por tan solo $10 €$ he conseguido una magnífica hamburguesa. Suponemos, por ello, que que hasta ahora hemos sido eficientes pero cabe preguntarse, no obstante, si he disfrutado con su sabor, si he saciado mi hambre y me ha reportado fuerza y energía para seguir trabajando, esos eran los resultados esperados, no la hamburguesa en sí.

Es frecuente que periodistas o líderes políticos o sindicales argumenten sus críticas según los inputs. Un descenso en el gasto público dedicado a educación o sanidad basta para atacar de forma irrefutable la política educativa o sanitaria. Menos necio, aunque también lo es, es justificar la crítica basándose en los outputs y criticar por ejemplo que se cierren oficinas de atención a los consumidores o un centro de formación de profesorado, o que se han celebrado menos cursos de protección a desempleados. La política educativa es uno de los ejemplos que mejor ilustra la crítica basada en inputs y no en outcomes. Tras investigar la relación resultados educativos (PISA y PIACC) con los recursos utilizados, la OCDE demuestra que a partir de cierto nivel de gasto no hay una correlación entre los resultados obtenidos y los recursos empleados. Solo el gasto inferior a $50.000 \$$ por estudiante de 6 a 15 años está positivamente relacionado con el rendimiento y la equidad. Una vez alcanzado este nivel de gasto, la relación entre el gasto en educación y el rendimiento deja de existir. El gasto acumulado en España en un alumno desde que tiene 6 años hasta que tiene 15 es de 82.178 dólares. Lo importante es cómo se gasta y no cuánto se gasta.

Lo que realmente hay que valorar, y escasamente son valorados, son los impactos de las medidas, el resultado de la política pública en cuestión. El objetivo, y el resultado (Serra, A. 2008), se definen por el grado de modificación que el gobierno y la acción pública se proponen lograr para una determinada variable social o, desde el punto de vista de la productividad, si se ha mejorado o mantenido un resultado con los mismos o menos recursos o productos; es decir, siguiendo aquellos ejemplos, si la protección del consumidor se ha visto reducida, si ha empeorado la preparación de los docentes o si la eliminación de determinados cursos a desempleados ha empeorado las cifras de desempleo.

Si bien en el caso del gasto productivo el riesgo de ineficiencia se da tanto en la generación de los outputs como en la evaluación del resultado, en el caso del gasto redistributivo el peso de la ineficiencia se centra en la relación entre el importe destinado y el impacto buscado pudiéndose producir dos tipos de ineficiencias (que suelen ir unidas): de volumen y de distribución. Las ineficiencias de volumen se producen cuando un aumento en la cuantía de la transferencia no implica una mejora en el resultado o en el impacto 4 . Las ineficiencias de distribución pueden producirse por el abuso o fraude de los destinatarios ${ }^{5}$ y por la aplicación del criterio de la universalidad ${ }^{6}$ en vez de un criterio residual.

\section{d) Las externalidades}

Volvamos a la hamburguesa: Ya casi la he terminado y empiezan los remordimientos; si esa hamburguesa está tan rica algo malo ha de tener: ¡Maldito colesterol!. El colesterol representa los efectos indirectos o colaterales perjudiciales, aunque también podrá haber externalidades positivas, como el beneficio obtenido por el dueño del restaurante. Algo parecido ocurre en la implementación de las políticas públicas. Se plantea nuevamente el debate "Hayek contra Keynes", entre quienes demuestran que las externalidades que implica el gasto público son preferentemente negativas (pérdida de competitividad, pérdida de ahorro y gasto productivo privado... ) y quienes fomentan la intervención pública y la contratación de funcionarios como soluciones a la recesión económica y al paro.

Bastiat explicaba en 1839 los perversos efectos de la intervención pública en sus artículos "Lo que se ve y lo que no se ve" que introduce del siguiente modo: "En la esfera económica, un acto, una costumbre, una institución, una ley no engendran un solo efecto, sino una serie de ellos. De estos efectos, el primero es sólo el más inmediato; se manifiesta simultáneamente con la causa, se ve. Los otros aparecen sucesivamente, no se ven; bastante es si los prevemos. Toda la diferencia entre un mal y un buen economista es ésta: uno se limita al efecto visible; el otro tiene en cuenta el efecto que se ve y los que hay que prever. Pero esta diferencia es enorme, ya que casi siempre sucede que, cuando la consecuencia inmediata es favorable, las consecuencias ulteriores son funestas, y vice-

4 El cheque bebe, el Plan E, etc.

5 Cualquiera que gestiona becas conoce el riesgo de fraude debido a la economía sumergida que provoca que los peticionarios aporten certificados de IRPF de ausencia de ingresos mientras que otros que declaran mínimos no obtienen la beca; es decir, pierden la beca aquellos que la merecerían en favor de otros que no la merecen.

6 Nuevamente, el cheque bebé es un ejemplo, o la rebaja en el precio de comedor escolar a todas las familias independientemente de sus ingresos (en alguna Comunidad autónoma se subvenciona el precio del comedor escolar mediante transferencia desde la Administración a los centros o a las empresas para que las familias -todas, independientemente de su nivel de renta- paguen menos). 
versa. Así, el mal economista persigue un beneficio inmediato que será seguido de un gran mal en el futuro, mientras que el verdadero economista persigue un gran bien para el futuro, aun a riesgo de un pequeño mal presente". Bastiat hace que nos preguntemos si los beneficios generados por las intervenciones públicas son superiores a los costes (directos y externalidades) que comportan y en muchas ocasiones "vemos" que sí porque, precisamente, "no vemos" esas externalidades negativas ocultas.

En efecto, investigaciones sobre la relación entre tamaño del sector público y crecimiento (Wolf, 1987) ponen de manifiesto que, por cada 10\% de aumento del peso del sector público -medido por la relación existente entre gasto público y producto total- la capacidad de crecimiento anual se reduce de 1 a 4 puntos). Sin embargo, Europa sigue convencida de que la solución al crecimiento son los planes de estímulo y políticas de demanda, como el "Plan E" de Zapatero.

\section{INEFICIENCIAS COYUNTURALES O ESTRUCTURALES: ¿HAY SOLUCIÓN?}

Pero, ¿son esas ineficiencias coyunturales?, ¿es posible mejorar la gestión y ser más eficiente?. El gran problema de la gestión pública es que, per se, es estructuralmente ineficiente. Quizá las nuevas tecnologías, cambios en procesos, etc, puedan mejorar los resultados y la eficiencia; pero ello será en términos relativos (respecto a otra Administración o a otro intervalo temporal) pero no con carácter absoluto. Múltiples causas, que se describen a continuación, forman un sistema de imposible ruptura, que hace que la toma de decisiones y la gestión no puedan llegar a ser eficientes.

\section{a) El político no prefiere la eficiencia: los factores "político-económicos"}

La teoría de la elección pública (Wicksell, Buchanan y otros) es una de las formulaciones más claras explicativa de los fallos estructurales del sector público que concluye que la provisión de bienes y servicios públicos no sigue criterio alguno de eficiencia en la asignación de recursos puesto que el mercado es un mercado político en el que los criterios de racionalidad están ausentes. En relación con esta teoría se dan dos fenómenos en el escenario político, señalados anteriormente: El primero de ellos podemos denominarlo el "efecto imitación o contagio", que induce a los líderes políticos a implementar las iniciativas que desarrollen sus homólogos de otros territorio, a "no ser menos". Ello explica que haya campus universitarios en todas las provincias, agencias de evaluación universitaria en varias comunidades autónomas, que tengamos 23 conservatorios superiores de música... El segundo es el ciclo político que fijan los procesos electorales, determinante de cualquier decisión y los retardos: el gasto público se incrementa porque la proximidad del periodo electoral induce a políticos y burócratas a conceder beneficios para intentar repetir su voto lo que trae más ineficiencia: las economías actuales se ven abocadas a sufrir déficit fiscales continuados como consecuencia de la espiral electoral y su frecuencia.

En resumen: el objetivo político o electoral a corto plazo prima sobre la eficiencia; entre eficiencia o votos, el político prefiere los votos. El ex primer ministro de Luxemburgo Jean-Claude Junker, no podía ser más sincero cuando en 2007 decía "Todos sabemos qué hacer, pero no sabemos cómo ser reelegidos una vez que lo hemos hecho".

\section{b) El gestor no puede: factores “jurídico-administrativos"}

A estos factores "políticos" se unen otros factores "jurídicos" que se justifican en la necesidad de garantizar fines socialmente deseables como la igualdad en el acceso al empleo público o entre los proveedores de la Administración y evitar prácticas corruptas. Sin embargo, aun siendo necesarios son también grandes generadores de ineficiencia.

Alejandro Nieto lo explicaba de forma muy clara en su clásico, y lamentablemente también actual, "La organización del desgobierno”: En primer lugar, advertía de la desorbitación del principio de legalidad: cualquier cosa tiene que ir precedida de una norma y ha de publicarse... "se ha formado una competitividad peculiar... el prestigio de un ministro se mide por su capacidad de lograr la aprobación de leyes en las Cortes y decretos en el Gobierno, sin que nadie se preocupe luego de la operatividad de tales disposiciones". En segundo lugar, los farragosos modelos de contratación y gasto: Las características del procedimiento de gasto son la lentitud, la inutilidad en relación a su fin (no evitan la corrupción ni el despilfarro) y la criminogeneidad pues provocan situaciones en que el delito es casi obligado, "Según sabemos, dice Nieto, nada puede gastarse si no hay consignación presupuestaria ni pagarse si no se ha realizado la prestación... sea como fuere, la Administración siempre sale perdiendo: porque el servicio se retrasa, o no funciona o funciona mal. Y el día en que los interventores y directores, escarmentados, se decidan a cumplir la ley, la administración quedará paralizada". Y, finalmente, no podía faltar, el sistema de empleo público como elemento multiplicador de la ineficiencia cuyas normas de selección y de carrera administrativa han generado un funcionariado preparado pero desmotivado y desaprovechado a causa de la injerencia política, sindical y la ausencia de un sistema de recompensas y sanciones eficaz. 
Las pautas del comportamiento burocrático (Frey, 1987) provocadoras de ineficiencia e inevitables con el actual sistema son, entre otras, las siguientes: (i) El bajo o nulo incentivo que tienen los funcionarios para actuar de una manera socialmente óptima ya que, salvo excepciones, no existe la responsabilidad individual o su desempeño no tiene consecuencias retributivas o de carrera. (ii) Los aparatos burocráticos que se quedan sin funciones desaparecen difícilmente, al no haber un test de eficacia y tener "mala prensa" el cierre de unidades u órganos Administrativos. Y (iii) la tendencia o inercia a ampliar o cubrir el número de puestos internos de trabajo (especialmente en Administraciones pequeñas donde puede tener rédito electoral).

Y esto no es exclusivo de España, como expresa el Informe Closets de 1989, «los gestores no tienen más remedio que escoger entre la ineficacia o la irregularidad. Ineficacia cuando se someten a reglas detalladas, a procedimientos confusos y poco adaptados. Irregularidad cuando se rebelan e intentan eludir las reglas»... La aplicación asfixiante de los procedimientos horizontales ha conducido a la crisis de responsabilidad que sufren las unidades prestadoras de servicios en la Administración pública”. En este diagnóstico coincide la Efficiency Unit en 1988: “En el momento presente la libertad del funcionario individual para gestionar de un modo eficaz y responsable en la Administración está gravemente limitada. No sólo existen controles sobre los recursos y objetivos, como debe haber en cualquier buen sistema, sino en la forma de gestionar los recursos. [... ] Las reglas, por lo tanto, se conciben principalmente como una limitación y no como un apoyo; y de ningún modo como un incentivo para que los funcionarios gestionen de forma eficaz...". El informe francés llega a conclusiones similares al afirmar que el objetivo es «pasar del Estado del procedimiento al Estado de la responsabilidad», ya que, según su opinión: “La inflación de normas cada vez más complejas y precisas [... ] tiende a aniquilar la responsabilidad e iniciativa de todos los peldaños de la vida administrativa, lo que reduce considerablemente la eficacia de la acción pública" (Echebarría, 2008).

En definitiva, (Nieto 1984): “... el permanente desgobierno de España no es consecuencia de la incapacidad de los gobernantes ni resultado tampoco de su régimen jurídico determinado, sino que se trata de algo mucho más sencillo y, al tiempo, infinitamente más grave: entre nosotros, la res publica está organizada de una manera tal que hace inútiles los esfuerzos individuales de los directores más competentes e incluso las decisiones mejor intencionadas de los partidos políticos que ocupan el poder. Por así decirlo, se ha montado una excelente organización del desgobierno. O lo que es lo mismo: con estas cartas y con estas reglas de juego, no hay jugador por bueno que sea, que pueda ganar la partida. Para prosperar habría que alterar las condiciones del "sistema"; pero la naturaleza de éste -y aquí viene lo desperanzado de la situación- obstaculiza gravemente su propia modificación.”

\section{c) El ciudadano no sabe: Factores socio-culturales}

Se ha descrito hasta el momento cómo el político tenderá a priorizar los criterios y plazos electorales sobre los criterios técnicos o económicos y cómo el gestor, maniatado por los sistemas públicos de empleo, de contratación y de gasto, por mucho que se implique, difícilmente conseguirá ser eficiente. Pero, tristemente, tampoco se advierte que haya una presión social que premie (electoralmente) o fomente el cambio de sistema. De este modo el círculo vicioso se consolida.

Y ello es, en parte, por la ignorancia sobre el coste de los servicios públicos o la idea de que "el dinero público no es de nadie"7 y esa falsa creencia o la ignorancia del coste real de los servicios o bienes públicos está generalizada incluso entre quienes se les supone unos mayores niveles de información y análisis. Así lo demostraba el resultado de una encuesta (Perez-Díaz y Rodríguez, 2015), que indicaba que apenas un 10\% de los estudiantes universitarios conocía lo que costaba, de forma aproximada, la docencia que recibía, porcentaje que no alcanzaba el $7 \%$ en la población en su conjunto.

Un segundo factor de la inacción política para frenar el intervencionismo es la oposición social a las reformas por la "mala prensa” que tiene oponerse a ciertas políticas públicas. Es "políticamente incorrecto” defender la austeridad o mostrarse contrario a determinadas subvenciones. Bastiat lo explicaba así: “¿Saben de qué se acusa a los economistas?: De, cuando rehusamos la subvención, rechazar la cosa misma que se subvenciona. ¿Qué pensamos que el Estado no debe subvencionar a los artistas? Somos unos bárbaros que juzgamos las artes inútiles".

La dependencia del Estado y la preferencia por lo público también es provocada por otros factores como la falta de cultura sobre el aseguramiento, especialmente sobre el aseguramiento financiero, por la disociación entre los ingresos y gastos públicos y por la inmediatez de los efectos de las políticas de equidad y la lejanía de los efectos de las políticas liberalizadoras. Recordando a Tocqueville, las ventajas que implica la igualdad eran evidentes mientras que «los bienes que proporciona la libertad sólo aparecen a largo plazo». Estos factores favorecen una conciencia social que atribuye la responsabilidad del Estado sobre los problemas individuales. En

7 Carmen Calvo, Exministra de Cultura: “Estamos manejando dinero público, y el dinero público no es de nadie.”. En entrevista ABC 29 de mayo de 2004: http://www.abc.es/hemeroteca/historico-29-05-2004/abc/Home/carmen-calvo-en-mes-y-medio-no-se-puede-montar-unministerio-que-no-existia_9621740957990.html. 
efecto, según muestra el Centro de Investigaciones Sociológicas (CIS, 2014), ante la pregunta sobre la preferencia entre pagar más o menos impuestos y obtener más o menos mejoras en los servicios público, en una escala de respuestas de o a 10, en que o representa a quienes están completamente de acuerdo con la preferencia "mejorar servicios públicos aunque haya que pagar más impuestos" y 10 los que prefieren "pagar menos impuestos aunque haya que reducir servicios públicos" el $32.2 \%$ se situaba en el 5 mientras que en los valores o a 4 se situaba el $36.3 \%$ y en los valores 6 a 10 el 21\%. También, sobre la responsabilidad del Estado (CIS, 1996), un 46.3\% consideraba que "El estado es responsable de todos y cada uno de los ciudadanos, y tiene la obligación de ayudarles a solucionar sus problemas", un 35.7 que "El estado solo es responsable del bienestar de los ciudadanos más desfavorecidos" y solo un $13 \%$ que "Los ciudadanos son los verdaderos responsables de su propio bienestar y tienen la obligación de valerse por si mismos para solucionar sus problemas.

\section{d) La sociedad no ayuda: Otros factores sociopolíticos}

Existen otros elementos que no solo impiden romper o siquiera cuestionar el intervencionismo social y culturalmente consagrado sino que contribuyen a su asentamiento: el sistema educativo, el papel de los medios de comunicación y el "activismo judicial".

Decía Leibniz: "Háganme maestro de enseñanza y me encargo de cambiar la faz del mundo". Es cierto que la gran base social y política que compone el sistema educativo en general y el profesorado en particular, está condicionada por su dependencia del sector público y altamente sindicalizada lo cual determina su posición ante ciertos temas. ¿Se imagina un profesor planteando un debate de forma neutral ante sus alumnos de secundaria sobre el cheque escolar?, Evidentemente, no. Pero sin embargo cuentan con textos en libros de Educación para Ciudadanía para abominar (subliminalmente o no) del capitalismo y del libre mercado, como en los extractos siguientes (Lacalle, 2015) “... lo que está fuera de control es, precisamente, el capitalismo, y el socialismo no es otra cosa que el freno de emergencia. Es la única esperanza que le queda a la humanidad para pararle los pies al capitalismo" (Ed. Serbal), "Es el Estado quien tiene la responsabilidad de garantizarnos los derechos y bienes más vitales a lo largo de nuestras vidas" (Agaida, Pearson Educación Alhambra) y "Esta teoría (por el neoliberalismo) resulta funesta para las economías débiles, para los obreros en general, y en especial para los más pobres" (Mc Graw Hill, Abad, 2010).

Pero tanta influencia como el sistema educativo o más ejercen los medios de comunicación. Cualquiera que haya trabajado cerca del poder político conoce el sesgo predominante entre los profesionales de la información, favorable a la intervención pública. Lo que vende es la queja y la demanda ilimitada de servicios y resaltar los fallos del mercado en vez de los del "no mercado".

Finalmente ha de destacarse un elemento que impide romper el asentado intervencionismo: el activismo judicial y el papel de reguladores que algunos tribunales de justicia asumen. El índice de Libertad Económica de España referido a 2015 analiza algunos casos en los que tribunales españoles han actuado de facto como legisladores, por lo general en contra de los principios básicos de la economía de mercado. Estamos ante lo que en la literatura económica suele denominarse "regulación mediante litigación"; los jueces pueden, en concreto, establecer condiciones o aplazar, a veces durante largo tiempo, la entrada en vigor de reformas económicas o, simplemente, hacerlas imposibles de llevar a la práctica". Para ello dicho Informe pone varios ejemplos de otros países y casos ocurridos en España en el ámbito laboral o administrativo (como el caso de la suspensión de un programa de gestión privada de determinados servicios hospitalarios, que habría mejorado la eficiencia).

La solución, por tanto al problema de la ineficiencia, es evitar las causas que la originan, los factores descritos pero ¿es ello es posible?. ¿Alguien cree que es posible que el político no esté condicionado por los plazos y los criterios electorales?, ¿alguien cree que es posible un modelo de empleo y de gasto en el sector público que garantice la eficiencia y al mismo tiempo evite las prácticas corruptas?, ¿alguien cree que la sociedad dejará de demandar más y más prestaciones?. La respuesta a las tres preguntas es, evidentemente, no, no y no. El triángulo de hierro formado por los burócratas, los grupos de interés que se benefician económicamente de las políticas puestas en marcha por la anterior Administración y los miembros del partido que priorizan las políticas clientelares sobre las reformas estructurales, acaba por imponerse, manteniendo el status quo y frustrando el cambio (Friedman, 1984).

\section{LA DIMENSIÓN DEL PROBLEMA: REMANGARSE O LOS MONOS DE GIBRALTAR}

El Informe CORA señala que la Administración equivale, en términos de gasto público, a la mitad de la economía, "si una de las variables estratégicas para el desarrollo económico es el aumento de la competitividad, una organización que equivale a cerca del 50\% del PIB debe ser competitiva”. Pero ojalá solo fuera un problema de competitividad y de crecimiento; es también un problema de sostenibilidad, de pervivencia del EB. 
Podemos seguir como los monos de Gibraltar, taparnos los ojos y no ver la gravedad de la situación de déficit continuados, taparnos los oídos y no escuchar los evidentes datos de envejecimiento, y taparnos la boca para no proponer soluciones reales. Podemos pensar que el sector público puede llegar a ser eficiente a pesar de su tamaño. Podemos, en definitiva, querer ganar las elecciones y hacer caso omiso de las certezas ampliamente aceptadas entre los economistas (Requeijo, 1995): (i) Una economía de mercado no puede soportar un gasto público que suele rondar el 50\% del PIB. "La necesidad de recortar la intervención pública en la economía, reduciendo el gasto en términos relativos es la primera de las certezas que solo los intervencionistas recalcitrantes -los que, en suma, rechazan el mercado- pueden negar...". (ii) La certeza sobre la bola de nieve -déficit-deuda- también concita apoyos muy amplios y, en este sentido, la interpretación keynesiana del déficit ha quedado desvirtuada por la realidad: el déficit continuado no tiende a autocorregirse al crecer el producto y los ingresos fiscales; tiende a autoimpulsarse a través de su propia carga financiera ${ }^{8}$. $Y$ (iii) finalmente aparece como otra certeza la debilidad del Estado de Bienestar (dificultad de financiación por el paro y las tendencias demográficas)

El Estado de bienestar nació con una clara vocación intervencionista en reacción a la radical separación entre Estado y Sociedad propugnada por el Estado liberal. La construcción (y crecimiento imparable) del EB hasta nuestros días se explica por ésta ha sido guiada por tres premisas que han condicionado poderosamente las elecciones de política pública y de las prácticas administrativas: (i) "La separación de los ámbitos público y privado, que asumen responsabilidades distintas, si bien a diferencia del Estado liberal no se trata de una separación estricta, puesto que se otorga una clara primacía al Estado. En esta concepción, lo "público", entendido como la búsqueda del interés general, se considera patrimonio exclusivo del Estado"; (ii) La creencia en la bondad intrínseca de la intervención pública y en la capacidad del Estado para resolver los diferentes problemas sociales, causa de la autosuficiencia y el paternalismo asociados con el Estado de bienestar. Y (iii), "la convicción de que la producción directa por el Estado era y sigue siendo la mejor garantía y la manera más adecuada de hacer llegar a todos los ciudadanos los bienes considerados socialmente "preferentes" (como la sanidad, la educación o la cultura). Así, en la mayoría de países europeos, plantear la provisión de un determinado servicio público ha significado de manera automática que su prestación debía ser realizada por una organización pública... detrás de esta opción político-organizativa, que ha comportado un fuerte crecimiento del empleo público, se esconde una clara desconfianza hacia la sociedad civil y el mundo empresarial, a los que, por lo general, no se reconoce la capacidad suficiente para participar en la prestación de los servicios públicos o la motivación adecuada para actuar de acuerdo con los valores que deben regir la actuación pública". La consecuencia de las premisas anteriores "que la única manera de dar respuesta a unas necesidades sociales, siempre crecientes, no será otra que disponiendo cada vez de más recursos públicos...." (Mendoza y Vernis, 2008).

La deuda y la demografía señalan que la gobernanza en los países ricos tiene que cambiar (Micklethwait y Wooldridge, 2015). "En 2030, el 22\% de los ciudadanos del club de países ricos de la OCDE tendrán 65 años o más, casi el doble que el porcentaje de 1990. China alcanzará ese punto tan solo 6 años después. Una sociedad envejecida va a enterrar al Estado de dos maneras: en primer lugar, el coste de las pensiones va a aumentar enormemente; y la propagación de enfermedades crónicas pondrá una carga aún mayor sobre la parte menos eficiente del sector público; la atención de la salud.". Asimismo, el gobierno de EEUU solo ha tenido superávit en cinco ocasiones desde 1960; Francia no lo ha tenido ni una sola vez desde 1974-1975... En la vieja Europa la población en edad de trabajar alcanzó su punto máximo en 2012 con 308 millones y está previsto que descienda hasta los 265 millones en el año $2060 . .$. La tasa de dependencia de la vejez de mayores de 65 años respecto a las personas de entre 21 y 64 aumentará del 28 al $58 \% "$.

España no es ajena a estos problemas que se avecinan en el mundo desarrollado. En nuestro país "las Administraciones responsables de los servicios públicos fundamentales asociados al envejecimiento -pensiones, sanidad y dependencia- se enfrentarán a problemas de sostenibilidad financiera adicionales a los actuales en las próximas décadas, como consecuencia de las tendencias crecientes a largo plazo del gasto en pensiones y en cuidados de larga duración" (Pérez, Cucarella y Hernández, 2015)

\section{Y ENTONCES, ¿QUÉ?: LA TEORÍA DE LAS 3S}

La teoría $3 S$ es una teoría explicativa del proceso ordenado (sucesivo, no simultáneo) necesario para reducir la ineficiencia en el sector público. Las tres eses se refieren a las iniciales de las tres fases que debería seguir ese proceso de revisión y reducción: Supresión, Sustitución y Simplificación.

\section{a) Suprimir}

Debemos empezar preguntándonos qué preservar, qué mínimo debe asegurar el Estado y suprimir (institucional, territorial y funcionalmente) todo lo evitable: ¿son necesarias todas las instituciones existentes?, ¿pasará

8 Los defensores de Keynes deberían saber que éste sostenía que el Estado nunca debía gastar más de una cuarta parte del PIB. 
algo porque desaparezcan los 90 observatorios que CORA sugiere suprimir?, ¿necesitamos más de 70 universidades públicas?, ¿todo núcleo de población necesita una organización municipal?... Y desde esas cuestiones a otras más "administrativas": ¿es necesario simultanear ferrocarril y autobús entre los mismos municipios?, ¿es necesario el DNI para quien ya tiene carné de conducir?

La primera necesidad es, por tanto, reducir, mediante su completa supresión, aquellas funciones que no debe llevar a cabo, ni directa ni indirectamente, el sector público. No se trata de un asunto de dimensión sino de si la función, el órgano, o el ente, son prescindibles. La supresión, por tanto, puede ser institucional u orgánica por la cual se eliminaría directamente el órgano o ente al completo sin que ello afecte a ninguna de las funciones o prestaciones del Estado de Bienestar. Responden a este tipo de supresión las propuestas de eliminación de las figuras autonómicas del defensor del pueblo, consejos consultivos, observatorios, diputaciones provinciales, eliminación de entidades locales menores... Junto a ese tipo de supresión subjetiva o “del quién” podemos hablar también de una supresión objetiva o "del qué”: la supresión funcional o competencial. La tarjeta sanitaria es un ejemplo de ello. Para qué es necesaria si ya hay tarjetas identificativas oficiales (DNI por ejemplo) con las que podría cotejarse la pertenencia a la base de datos de ciudadanos con derecho a la asistencia sanitaria.

Otro tipo de supresión es la reducción de transferencias públicas (total o parcial), eliminando o disminuyendo subvenciones inútiles (por no provocar el outcome deseado) o prestaciones sociales (o sustituyendo dichas prestaciones por sistemas mixtos). En este sentido se plantea el tradicional debate sobre universalidad o selectividad en los modelos de Estado de Bienestar, siendo un modelo selectivo o residual hacia las personas "sin posibilidad de posibilidad" claramente más sostenible.

\section{b) Sustituir}

Suprimido todo lo prescindible debería analizarse si lo que el Estado desea prestar o fomentar ha de prestarlo o fomentarlo directamente o puede hacerlo (o ya lo hace) el sector privado por no ser necesario el ejercicio de autoridad pública. La sustitución de gestión pública por privada puede articularse de cuatro modos.

En primer lugar, mediante la "externalización de la gestión”, a través de múltiples fórmulas jurídicas existentes para ello como el contrato administrativo de gestión de servicios públicos (concesión de servicios, gestión interesada, concierto, sociedad de economía mixta), los partenariados público-privados, la concesión de dominio público, censos enfitéuticos, el derecho de superficie, etc.

Es frecuente que se alcen las voces en contra de los procesos de externalización argumentando que la autoridad pública no puede ser ejercida fuera de la Administración ni por quien no es funcionario. Sin nada que objetar a dicho argumento, en muchas ocasiones se observa que lo que se pretende no es salvaguardar dicho principio sino defender otros objetivos, también legítimos pero ajenos al bien común, como el mantenimiento de condiciones laborales o la fortaleza sindical. Aun no discutiendo que la autoridad pública debe ser ejercida desde la Administración y por funcionario público, sí que lo es la extensión de la condición de funcionario a cualquier empleado público (médicos, maestros, auxiliares administrativos, bomberos...) o la necesidad de que cualquier autorización haya de ser expedida por la Administración. Así nos encontramos con que quien enseña inglés en una Escuela Oficial de Idiomas es un funcionario y que es el Ayuntamiento quien te da la licencia para abrir una peluquería, mientras que de la autorización para circular con un vehículo pesado (la "ITV”) o de la auditoría de las cuentas de una empresa del IBEX se encargan las empresas privadas.

Ciertamente, los contornos no son claros y por ello debemos acudir de nuevo a una aplicación estricta del principio de subsidiariedad por el que la gestión directa de la Administración solo se justifique cuando sea necesario el ejercicio de la autoridad en sentido restrictivo (Administración de Justicia o actividad administrativa de policía) y sean funcionarios quienes han de tener garantizada su objetividad e imparcialidad (por ejemplo, las autoridades que dictan resoluciones o sus informes preceptivos, no cualquier nivel de la gestión previa o posterior).

Una segunda fórmula de sustitución es "prestación por financiación"; es decir, en lugar de responsabilizarse directamente la Administración de prestar el servicio, su función sería asegurar el acceso de la población a dichos servicios prestados por el sector privado. Ejemplos de ello es el llamado "cheque escolar", el "cheque universitario" o la posibilidad de que las Administraciones se desprendieran de sus residencias de ancianos o escuelas de idiomas y en cambio, ofreciesen ayudas o becas para residir o estudiar en dichos centros del sector privado.

Esta financiación, preferiblemente, no debería ser "positiva” mediante becas, ayudas o subvenciones; sino "negativa", mediante desgravaciones fiscales, lo que lleva a la tercera propuesta de sustitución: "Subvención por desgravación", que implica cambiar un efecto "asistencialista y paralizante” por un incentivo "dinamizador”. 
Finalmente, la cuarta fórmula de sustitución sería “demanda obligatoria por voluntaria” y, cuando ello no sea posible, "monopolio por competencia". Se trata de que el ciudadano pueda elegir entre disfrutar de la prestación/ cobertura o rechazarla y, en caso de que no pudiera ofrecerse tal elección, se debería poder elegir al prestador/ asegurador. Ejemplo de ello es la recepción (no elegida) de la señal de una televisión autonómica. En este caso podría ofrecerse (como se hace en algunos países) la posibilidad de elegir entre tener acceso a la conexión (mediante el pago de una tasa) o rechazar la conexión (con el consiguiente ahorro). En casos en que, por el contrario, la Administración no quiere "dar a elegir" porque interesa socialmente asegurar que toda la población pueda tener derecho a una determinada prestación o a su cobertura (sistema de pensiones o garantía de la asistencia sanitaria, por ejemplo), debería evitarse el monopolio impuesto por el sector público y poder elegir al prestador, como se hace en el modelo sanitario holandés -considerado el mejor del mundo-, en el modelo "MUFACE", que podría universalizarse, o mediante sistemas mixtos en el sistema de pensiones.

\section{c) Simplificar}

Finalmente, solo cuando, siguiendo el principio de subsidiariedad, estemos ante una actividad que deba ser realizada directamente por el sector público, por no ser suprimible o sustituible, es cuando debemos simplificar la norma o el proceso mediante el que se realiza esa actividad administrativa, sea sancionadora, redistributiva de transferencias o prestadora de bienes o servicios.

En el proceso de simplificación juega un papel fundamental la extensión del uso de las nuevas tecnologías. A propósito de este uso en particular, y de cualquier medida de simplificación en general, conviene señalar la importancia de aprovechar sus efectos (mediante la consiguiente reducción de unidades o de recursos humanos por ejemplo). A pesar de los esfuerzos realizados hasta la fecha (especialmente en la Administración del Estado) y de que es inevitable el camino hacia la administración electrónica, el avance tecnológico no va acompañado de una mayor eficiencia pues los costes de la inversión en su desarrollo no son compensados con ahorros de gasto corriente. "Nuestro Leviatan está tan fuera de sintonía con el espíritu de la era de Internet como lo estaba el Estado de principios de la época victoriana con la era del ferrocarril: absorbe enormes cantidades de recursos, pero fracasa al intentar sacar provecho de la productividad que proporciona la tecnología moderna..." (Micklethwait y Wooldridge, 2015).

\section{LOS INTENTOS FALLIDOS O INSUFICIENTES}

Bien porque, como los monos de Gibraltar, no se acepta el problema del tamaño del EB; bien porque los responsables políticos no se atreven a aplicar la teoría de las 3S, por el temido coste electoral; bien porque interesados colectivos (sindicales o funcionariales) construyen su "triángulo de hierro" friedmaniano que congela el status quo... ; por todo ello hacemos "lavados de cara", diseñamos Libros blancos, proyectamos planes de modernización o renovación administrativa o importamos técnicas gerenciales del sector privado como si fuera posible jugar al baloncesto en un campo de futbol. Pero, aun reconociendo que todo este trabajo de mejora pudiera tener éxito solo será "una tirita en una hemorragia”. ¿Por qué fallan o no son suficientes esas soluciones?

Al igual que con las "tres erres" para la protección medioambiental (Reducir-Reutilizar-Reciclar) el orden es fundamental. Simplificar sin antes sustituir o sustituir sin antes suprimir es el primer error. Como se explicaba anteriormente, la teoría de las $3 \mathrm{~S}$ es un proceso ordenado o jerarquizado de tres soluciones. ¿Para qué mejorar, por ejemplo, el proceso de expedición y distribución de la tarjeta sanitaria si podría eliminarse?, ¿para que esforzarnos en gestionar con calidad si el proceso es prescindible?. Y si la intervención pública no es prescindible, nos encontramos frecuentemente con otro fallo señalado en el apartado anterior: tras desarrollar con éxito fórmulas de simplificación mediante la extensión de la administración electrónica o mediante la simplificación normativa, no son aprovechados sus efectos con lo que lo que en principio podía ser un ahorro termina siendo un coste añadido.

Un segundo fallo, en el caso de la supresión institucional u orgánica es reducir sin suprimir olvidando lo que la experiencia demuestra: todo ente no extinguido vuelve a reproducirse. Si él ente es prescindible, al contrario de lo que suele suceder en la práctica, debemos valorar su existencia, no su dimensión.

Otros dos fallos que frecuentemente nos encontramos cuando algún político, tímidamente por lo general, decide ir en la buena dirección, son las reducciones lineales y la descoordinación territorial. Como describe el Informe CORA, el Gobierno del Partido Liberal en Canadá, tras ganar las elecciones en 1993, ante una situación de deterioro de las cuentas públicas, decidió afrontar una reforma profunda. ¿Qué ocurrió allí que no ha ocurrido en España?: Frente a una reducción lineal en las partidas del presupuesto, Canadá optó por llevar a cabo un estudio profundo sobre la importancia de las diferentes políticas y programas y realizar los ajustes en aquello que no era prioritario. De este modo se eliminaron completamente o se modificaron (al alza o a la baja) las distintas partidas. Al mismo tiempo, las provincias canadienses se embarcaron en procesos similares, lo que resultaba fundamental, 
por el alto grado de descentralización política y el elevado gasto de los gobiernos regionales, que prestan la mayoría de los servicios al ciudadano.

Otra propuesta teórica para mejorar la eficiencia es el federalismo competitivo, fórmula que logra una mayor eficiencia ya que dificulta la duplicidad del gasto "porque se produce una clara delimitación de competencias y responsabilidad. La proximidad de los ciudadanos y su mayor capacidad de control de la Administración constriñe las posibilidades de expansión de las organizaciones burocráticas; y la oferta de bienes y servicios públicos se ajustará mejor a las demandas diferenciadas territorialmente de los ciudadanos de cada jurisdicción" Sin embargo, no hay una base empírica suficiente que pruebe que el tamaño del sector público varía inversamente con el grado de descentralización fiscal; numerosos estudios empíricos realizados no ofrecen evidencias sólidas en favor de la hipótesis de la descentralización, e incluso algunos la contradicen abiertamente (Corona y Puy, 1998).

El argumento de que la autonomía y la responsabilidad fiscal (tanto en ingresos como en gastos) conduciría, en cada territorio, a una adecuación de ingresos y gastos a las preferencias de los ciudadanos y a un ajuste competitivo entre las regiones favorecedor del crecimiento económico (al procurar los gobiernos que se "vote con los pies") no deja de tener su lógica; sin embargo, quienes ven en la descentralización un instrumento efectivo para controlar las tendencias expansivas del Leviatán ven frustadas sus expectativas cuando aparecen los vicios derivados de la cercanía del responsable político al residente-votante (el efecto imitación, la espiral de las promesas electorales, la teoría de la elección pública) que se imponen sobre las virtudes de esa relación, además de otros efectos negativos, como el menoscabo de la solidaridad entre las regiones o la fragmentación del mercado interior.

\section{CONCLUSIONES. DEL ESTADO DE BIENESTAR A LA SOCIEDAD DEL BIENESTAR}

La Administración es ineficiente intrínsecamente y esa ineficiencia, cronificada, no tiene solución. La preferencia del decisor político por el rédito electoral frente a la eficiencia sostenida, los modelos de empleo y gasto público que maniatan al gestor y otros factores socioculturales impiden una reforma eficaz del actual modelo.

Esa es la primera conclusión; la segunda: no cabe el Status quo de intervención y gasto y es imprescindible un cambio de modelo. Las soluciones aplicadas solo son leves mejoras que no evitan el riesgo de quiebra del sistema, riesgo que amenaza la propia sostenibilidad del EB y los derechos civiles y políticos.

Por ello (tercera conclusión) es necesaria una solución "radical"; es decir, que vaya a la raíz del problema, a cambiar el modelo. Y ello es lo que propone la Teoría de las $3 \mathrm{~S}$ que plantea un nuevo sistema en el que la subsidiariedad del Estado respecto a la sociedad civil sea real y efectiva. Para ello debe seguirse el proceso SUPRIMIR-SUSTITUIR-SIMPLIFICAR; un proceso ordenado y sucesivo de reflexión y "adelgazamiento", competencial y funcional, que implica (i) que los Gobiernos se responsabilicen de menos competencias, (ii) que de aquellas que necesariamente se tengan que responsabilizar se encargue de su ejecución el sector privado y (iii) que aquellas que tengan que ejecutar directamente se hagan bajo normas y procesos más simples.

\section{A MODO DE EPÍLOGO: DEL ESTADO DE BIENESTAR A LA SOCIEDAD DEL BIENESTAR}

$Y$ esa propuesta no renuncia al Bienestar, pero sí a que la responsabilidad de ese Bienestar sea monopolizada por el Estado; el bienestar social hay que buscarlo por otro camino, no por el de la demanda ilimitada a la niñera omnipresente en que se ha convertido el Estado sino por el de individuos y entidades privadas responsables de su propio interés y del interés social. El Estado ha arrebatado de su responsabilidad a la sociedad civil y ésta debe recuperarlo. El sector público no "es" sino que "se hace"; individuos, asociaciones, familias o empresas han de recuperar la res publica que les pertenece y ejercer su responsabilidad directa en lo público. El Estado "no debe, en principio, dar al hombre lo que necesita para asegurarse el bienestar, sino darle la seguridad de que por sí mismo puede ganarse el bienestar que necesita, espoleando en él, con los adecuados incentivos, el ímpetu para abrirse camino en la vida, es decir, fomentando la responsabilidad de forjar la propia existencia, generando en el individuo la garra suficiente para afrontar la lucha con vistas a la realidad presente y a las eventualidades del futuro. $O$ sea, propiciando todo lo que el EB ha destruido, pretendiendo dar a todos una excesiva y, por ello, paralizante seguridad" (Termes, 1997).

En definitiva, se trata de equilibrar el trade off de responsabilidades: de un lado, la responsabilidad social "activa" (de hacer) por parte del sector privado y del llamado "tercer sector"; y, por otro lado, la responsabilidad "pasiva" (de no hacer) del Sector Público, que implica una autolimitación en sus funciones y en su gasto.

En relación con la mayor responsabilidad "activa" de la sociedad civil, han de potenciarse en ese proceso "suprimir-sustituir" todas las fórmulas ya existentes de colaboración público-privada y de partenariado social. Desde el voluntariado social hasta la participación de empresas, ONGs, fundaciones, etc en la prestación de servicios públicos y en la consecución de logros sociales, bien por su propio interés u objeto social, bien por cuenta del Sector 
Público, a través de las distintas fórmulas de colaboración (convenios, contratos de gestión de servicios y otras). Este nuevo reparto de roles, tareas y responsabilidades entre Estado, mercado y sociedad civil, distinto al que ha caracterizado al EB es el que plantea "el pluralismo de Bienestar" o también, más recientemente, el llamado "Estado relacional". Como explican Mendoza y Vernis (2008), podemos hablar también de lo que algunos han denominado la publicización de la sociedad (Bresser-Pereira) y otros la privatización del Estado (Savas). "La dicotomía público-privado ha llevado, históricamente, a identificar lo público con el Estado y lo privado con la sociedad civil. Simplificación que comporta otorgar al Estado el monopolio de lo universal, a identificar lo público con lo político y a negar a la sociedad civil su fuerte potencial de universalismo y solidaridad (Cortina)". El Estado liberal y el EB han constituido los dos grandes modelos de referencia en las sociedades democráticas, "Sin embargo, desde finales de los años ochenta existe una conciencia creciente de que es preciso encontrar nuevos modelos de Estado y de Administración que permitan hacer frente a los complejos problemas con los que nuestras sociedades se adentran en el siglo XXI". Una opción en esa búsqueda, continúan los autores citados “consiste en la transformación en profundidad de la lógica de actuación y de los mecanismos de intervención del Estado de bienestar, pero manteniendo sus principios inspiradores de universalidad y cohesión social. O dicho en otros términos: lo que habría entrado en crisis sería la manera en que históricamente se ha construido el EB, pero no la idea misma de una sociedad de bienestar en la que el Estado desempeña un papel significativo en la consecución de sus objetivos centrales".

Pero decíamos que para potenciar esa responsabilidad "activa” y esa "Sociedad del Bienestar" es necesario, por otro lado, una mayor responsabilidad "pasiva” por parte del sector Público, en primer lugar, fiscalmente, mediante la limitación de la capacidad impositiva y deudora y del gasto público. Aunque el modelo que propone el federalismo competitivo no garantice una solución al problema de la ineficiencia, sí que alguno de sus elementos puede contribuir de forma positiva: una mayor responsabilidad fiscal (y consiguiente descentralización) especialmente en materia de ingresos y la necesidad de que los poderes fiscales estén claramente delimitados en el texto constitucional son elementos clave en la búsqueda de la eficiencia. En esa dirección van también las autolimitaciones constitucionales, desde la Propuesta Número 13 de California ${ }^{9}$ al artículo 135 de la Constitución española que impone cuestiones tan criticadas como elementales para evitar los déficit fiscales continuados, como el principio de estabilidad presupuestaria, la prohibición de incurrir en un déficit estructural superior al establecido por la Unión Europea, la necesidad de autorización por ley para emitir deuda pública o contraer crédito o la limitación del volumen de deuda pública.

En definitiva, como constatan todos los autores citados e invita el presente artículo, si queremos preservar el bienestar, debemos "reinventar" la Administración pública; el reto político de la próxima década será, como se ha señalado "el de reparar y reformar los sistemas de gobierno". TH Marshall sostenía que los ciudadanos habían adquirido nuevos derechos en tres oleadas sucesivas: derechos civiles en el siglo XVIII, derechos políticos en el siglo XIX y derechos sociales en el siglo XX. El desafío del Siglo XXI es asegurar su sostenibilidad y evitar que la crisis económica y sus efectos políticos puedan poner en riesgo los derechos civiles y políticos. Un proceso de supresión-sustitución-simplificación derivado de una reflexión responsable sobre el papel y el alcance del Estado podrán conducir a ello; lo contrario, a la regresión de los derechos y bienestar alcanzados.

\section{REFERENCIAS BIBLIOGRAFICAS}

Cabrillo, F., Rocío Albert, R. y Biazzi, R. (2015). Libertad económica en España 2015 http://www.civismo.org/es/ actividades/indice-de-libertad-economica-en-espana-2015.

CIS (1996) Estudio 2.213 y (Julio 2014). Opinión pública y política fiscal (XXXI) Estudio n. 3034.

Comisión para la Reforma de las Administraciones Públicas (Informe 21/06/2013). http://www.seap.minhap.gob. es/dms/es/web/areas/reforma_aapp/INFORME-LIBRO/INFORME\%2OLIBRO.PDF.

Cuadrado Roura, J. R. Dtor. (2000). Política económica Objetivos e instrumentos. 2. ${ }^{a}$ Mc. Graw Hill Págs. 110 y ss.

Echebarría, K. (2008) “Los dilemas del diseño organizativo en la gestión pública”, en Los escenarios de la gestión pública del Siglo XXI. Francisco Longo y Tamyko Ysa (eds.) 2008. Escola d’Administració Pública de Catalunya.

Frédéric Bastiat, F. (1839). Ce qu'on voit et ce qu'on ne voit pas Disponible en castellano en http://bastiat.org/es/ lqsvylqnsv.html.

Frey B. S.: Para una política económica democrática, Alianza, Madrid, 1987, págs. 131-132. Citado en Cuadrado Roura, J.R. Dtor. (2000). Política económica Objetivos e instrumentos. 2. ${ }^{a}$ Mc. Graw Hill.

Friedamn, M. (1984). La tiranía del Status Quo.

Johnson, N. (1987). El Estado de Bienestar en Transición. La teoría y la práctica del pluralismo de bienestar. Ed. Ministerio de Trabajo y Seguridad Social, 1990.

Juan Corona J. y Puy P. (1998). Federalismo competitivo y financiación autonómica.

9 En 1978 fue la primera autolimitación fiscal aprobada tras las llamadas "rebeliones fiscales" en EEUU que se extendió a otros Estados y, en 1981 siguieron más de la mitad habían aprobado limitaciones en los impuestos o en el gasto. 
King, A. (1975). Overload: Problems of Governing in the 1970s Political Studies, vol. 23, nums. 2 y 3. Pag 282. Citado en Cuadrado Roura, J. R. Dtor. (2000). Política económica Objetivos e instrumentos. 2. ${ }^{a}$ Mc. Graw Hill. DOI: 10.1111/j.1467-9248.1975.tbooo68.x.

Lacalle, D. (2015). Acabemos con el Paro. Deusto, 2. ${ }^{a}$ edición.

Marshall, T. H. "Ciudadanía y clase social”, en T. H. Marshall y T. Bottomore. Ciudadanía y clase social, P. Linares (trad.), Madrid, Alianza, 1998.

Mendoza X. y Alfred Vernis A. "El Estado relacional y la transformación de las administraciones públicas" en Los escenarios de la gestión pública del Siglo XXI. Francisco Longo y Tamyko Ysa (eds.) 2008. Escola d’Administració Pública de Catalunya.

Micklethwait, J. y Wooldridge, A. (2015). La cuarta revolución. La Carrera global para reinventar el Estado. Galaxia Gutenberg.

Moreno, L. (2000). Ciudadanos precarios. Ariel sociología.

Nieto, A. (1984). La organización del desgobierno. Ed. Ariel.

Offe, C. (1994). Contradicciones en el Estado de Bienestar, Alianza Universidad.

Pérez F., Cucarella V. y Hernández L. (2015). Servicios públicos, diferencias territoriales e igualdad de oportunidades. Fundación BBVA e IVIE (Instituto Valenciano de Investigaciones Económicas)

Pérez-Díaz V. y Rodríguez, J. C. (2015). Opiniones de los Españoles sobre sus Universidades: Algunas perspectivas. Studia XXI y Fundación Europea Sociedad y Educación.

PISA 2012. Presentación Ministerio de Educación, Cultura y Deporte. http://www.mecd.gob.es/dctm/inee/ internacional/pisa2012/presentacionpisa2012.pdf?documentld=0901e72b81787b13.

Príncipe Guillermo de Holanda. Discurso de septiembre de 2013. Speech 17-09-2013 http://www.royal-house.nl/ documents/speeches/2013/09/17/speech-from-the-throne-2013 acceso, marzo 2016.

Serra, A. "La gestión para resultados en las organizaciones Públicas", en Los escenarios de la gestión pública del Siglo XXI. Francisco Longo y Tamyko Ysa (eds.) 2008. Escola d’Administració Pública de Catalunya.

Termes, R. (1997). “Del Estado de Bienestar a la Sociedad del Bienestar”. En Cuadernos de Sociedad. 8. Conferencia. http://web.iese.es/RTermes/acer/acero7.htm.

Wolf (1987). Markets of governments. MIT Press Cambridge, 1987. Citado en Cuadrado Roura, J. R. Dtor. (2000). Política económica Objetivos e instrumentos. 2. ${ }^{\mathrm{a}} \mathrm{Mc}$. Graw Hill. 